\title{
NICHT-NYSTAGMISCHE SPONTANE PATHOLOGISCHE AUGENBEWEGUNGEN
}

\author{
Eiji Sakata, Ru-Pei Lee, Yasuo SaKaki und Masashi Wada
}

\section{VORWORT}

Bei den Erkrankungen des Zentralnervensystems (ZNS), besonders bei den verschiedenen Erkrankungen des Diencephalons oder im infratentorialen Gebiet spielt es klinischdiagnostisch oft eine wichtige Rolle, die Eingenschaften der Augenbewegungen gründlich und sorgfältig zu analysieren und zu untersuchen.

Jedoch bisher haben die Forscher fast ohne Ausnahme ihre Aufmerksamkeit hauntsächlich auf Nystagmus gerichtet, und infolgedessen gibt es nicht wenige Referate über "Nystagmus und dessen Herdlokalisation" ${ }_{6,12,14,15,16,17,18,19,20,21}$.

Einerseits wurden oft die spontanen pathologischen Augenbewegungen außerhalb des Nystagmus nur als "Augenzitterung" oder "Flimmernphänomen der Augen" abgetan, ohne zu unterscheiden oder zu analysieren, ob diese Erscheinungen physiologisch oder pathologisch sind.

Deshalb unter den gegenwärtigen Verhältnissen sind die Referate über dieses Problem meistens hauptsächlich im Gebiet der Ophthalmologie bzw. Neurologie behandelt und diese bleiben doch nur fragmentarisch; es gibt keine einheitlichen Forschungen. ${ }^{2,3}$, $4,5,7,8,9,10,11,13$

In den letzten zwei Jahren förderten wir die Untersuchungen dieses Problems mit großem Interesse und erwarben die interessanten Kenntnise über den Zusammenhang mit dessen Pathophysiologie und Herdlokalisation, so möchte ich sie hiermit berichten und zur Information und Kritik vorlegen.

\section{KRANKENFÄLLE SOWIE PRÜFUNGSMETHODE}

In den letzten zwei Jahren behandelten wir 1317 Patienten in unserer Klinik und im Ambulatorium der Otoneurologie (Neurootologie) des mit der Klinik in Beziehung stehenden Krankenhauses. Darunter hatten wir 123 Fälle, bei denen nicht-nystagmische spontane pathologische Augenbewegungen wie im folgenden Abschnitt erwähnt klar erkennbar waren. Davon wurden 101 Fälle pathologisch-anatomisch, oder durch Operation bzw. durch genaue neurologische Prüfungen diagnostiziert.

Um die Einzelheiten der Fälle zu nennen: 6 Fälle des Kleinhirnhemisphärentumors, 2 Fälle des Medulloblastoms, 3 Fälle des Hämoangioms in Kleinhirnhemisphäre, 3 Fälle des Hämatoms im Kleinhirnwurm, 4 Fälle der Blutung in Kleinhirnhemisphäre, 6 Fälle des Tumors des dritten Gehirnventrikels, 3 Fälle des Pinealoms, 3 Fälle des Parkinsonsyndroms, 15 Fälle der vaskulären Mesencephalonläsion, 15 Fälle der spino-cerebellären 
Degeneration, 1 Fall der basilaren Impression, 2 Fälle des Tumors des vierten Gehirnventrikels, 4 Fälle der Vergiftung des anti-konvulsanten Mittels, 1 Fall der Hirnstammencephalitis, 12 Fälle des traumatischen Halssyndroms, 4 Fälle der vertebro-basilaren Insuffizienz, 1 Fall der Syringobulbie, 1 Fall des sogenannten "Hirnstammepilepsie, 10 Fälle des kongenitalen Nystagmus, sowie 2 Fälle des Tectalsyndroms des Mesencephalons.

Die Augenbewegungen von Blickkrampf sowie Konvergenzkrampf, deren Registrierung elektrisch unmöglish sind, wurden durch Kinetoskopie dargestellt, und sonst wurden NY22-Typ mit vier Phänomen ENG von Firma Lyon bzw. Biograph 180 System mit zwei Phänomen von Firma Sanei-Sokuki gebraucht.

Die Zeitkonstante wurde auf 3 Sekunden (Originalwellenform) sowie auf 0.03 Sekunde (Differentialwellenform) eingestellt, und bei der horizontalen Ableitung wurde die differente Elektrode am äusseren Augenwinkel, die indifferente Elektrode am Vorderhaupt angelegt. Bei der vertikalen Ableitung wurden die differenten Elektroden je am Obere der Augenbraue sowie am Untere des Augenlides angelegt.

Um die Einmischung des Kunstprodukts zu vermeiden, liessen wir die Patienten den Mund leicht öffnen. Ferner beobachteten wir die Augenbewegungen der Patienten und gleichzeitig verglichen wir sie mit der Registrierung, damit die Einmischungen von EMG als Augenbewegungen nicht falsch aufgefaßt werden. Darauf wurde auch achtgegeben, ob bei der vertikalen Registrierung EMG nicht erkennbar ist. Ferner mit Rücksicht auf die Einmischung von EEG wurde bei einigen Fällen EEG gleichzeitig durchgeführt.

\section{EINTEILUNG DER NICHT-NYSTAGMISCHEN SPONTANEN PATHOLOGISCHEN AUGENBEWEGUNGEN}

Wie am Anfang dieser Abhandlung erwähnt, sind bei verschiedenen Erkrankungen des Diencephalons oder die im imfratentorialen Gebiet außerhalb des Nystagmus die pathologischen Augenbewegungen mit verschiedenen Eigenschaften erkennbar. Wenn man sich darunter nur auf die Krankheiten, die unverkennbar pathologisch sind, beschränkt, sind die Namen oder Behandlungen solcher Krankheiten je nach den Forschern verschieden und sie sind noch nicht systematisch geordnet und eingeteilt.

Tabelle 1. Die Klassifikation der nicht-nystagmischen, spotanen, pathologischen Augenbewegungen. (Sakata)

Nicht-nystagmische spontane pathologische Augenbewegungen (non-nystagmic spontaneous pathological eye movements)

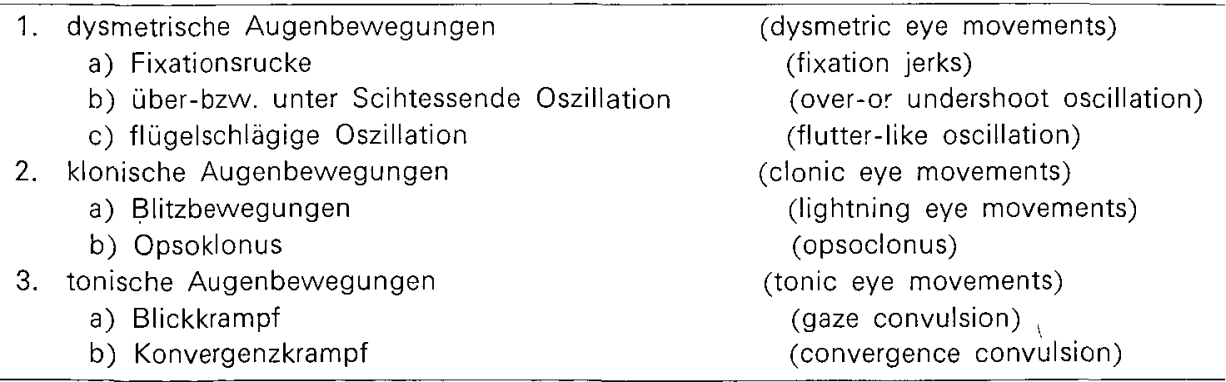


Wir halten es für richtig, auf Grund 1) des Entstehungsverhaltens (Umfang, Grad, Frequenz, zeitlicher Verlauf, Regelmäßigkeit) 2) Entstehungsbedingungen (ob sie bei Fixation erscheinen, oder beim Blick, Augenöffnen in der Dunkelkammer, oder beim Augenschließen) 3) Entstehungsmechanismus 4) Beziehung mit dem Herd der Erkrankungen 5) Einfluß der Arznei sie wie in der Tabelle I gezeigt einzuteilen und zu ordnen. Die Gründe dieser Gedanken werden im Abschnitt der zusammenfassenden Betrachtungen erörtert.

\section{PRÜFUNGSRESULTATE SOWIE KURZ ZUSAMMENGEFAßTE BETRACHTUNGEN}

1) Fixationsrucke, fixation jerks

Bei der Fixation bewegt sich der Augapfel des Menschen mehr oder weniger und diese Erscheinung wird mit der Zunahme des Alters immer auffallender. Die Erscheinung, bei der kein Spontannystagmus im engeren Sinne erkennbar ist, doch sich der Augapfel ruckartig orientiert schwingt, nennt man Fixationsrucke ${ }^{12}$ und sie ist bei den Hinterschädelgrubenerkrankungen nicht selten erkennbar. Außerdem richtet sich deren Ruckbewegung fast immer nach der wohlbefindenen Seite. Dazu hat sie die Neigung, auf der frühen Stufe der Erkrankung schon zu erscheinen, deswegen ist sie sehr bemerkennswert.

Wir registrierten diese Erscheinungen bei insgesammt 17 Fällen, d.h. einem Fall Kleinhirnhemisphärenabszess, einem Fall Medulloblastom, vier Fällen Astrozystom der Kelinhirnhemisphäre, zwei Fällen Mariescher Degeneration, einem Fall Spätatrophie der Kelinhirnrinde, sechs Fällen vaskulärer pathologischer Veränderung des Kelinhirns, einem Fall des Tumors des dritten Gehirnventrikels sowie einem Fall des Tumors des vierten Gehirnventrikels.

Das Bild zeigt den Fall des Medulloblastoms, bei dem die pathologischen Veränderungen beiderseits eingedrungen sind. Es ist aber auch bemerkenswert, daß sich diese Richtungen der Fixationsrucke nach rechts oder links wechseln.

Diese ruckartigen Bewegungen sind jedoch auch am Wohlbefindenen erkennbar, wenn er die Augen schließt, oder schläft oder übermäßig angestrengt ist, und diese Tendenz ist bei ENG mit kurzer Zeitkonstante noch merklicher. Ferner da bei Fixation die schnellen Augapfelrucke nach rechts und links oder die treppenförmigen Bewegungen (Treppenrucke) sichtbar sind, ist es notwendig, sie von der Fixationsrucke zu unterscheiden, damit diese zwei Bewegungen, die auch physiologisch entstehbar sind, als pathologische Erscheinungen nicht aufgefaßt werden.

2) Über-bzw. unterschiessende Oszillation, over-or undershoot oscillation

Im allgemeinen ist es kaum denkbar, daß bei den Kleinhirnerkrenkungen, besonders bei den Störungen der Hemisphäre der Spontannystagmus im engeren Sinne, der auf den Unterschied zwischen den rechten und linken Seiten des Vestibularissystems zurückzuführen ist, erscheint. ${ }^{15,16,17,19}$

In diesem Fall entsteht die Erscheinung, daß der Augapfel transitorisch schwingend zum Stillstand kommt, wenn der Blickpunkt auf dem Wege, wieder zurückzukehren, frontal 
bzw. rechts und links je etwa am $10 \mathrm{Grad}$ plötzlich angehalten wird, nachdem ihm eine leichte Bewegungsbelastung gegeben wird, nämlich nach den zwei-oder dreimaligen Hinund Rückbewegungen etwa am 20 Grad rechts und links. Der Grund, warum in diesem Fall nicht auf dem Weg nach Seiten sondern erst auf dem Weg, zurückzukehren, der Blickpunkt plötzlich zum Stehen gebracht wird, liegt darin, es zu vermeiden, den blickdysmetrischen Nystagmus falsch zu verwechseln. Diese Erscheinung ist von verschiedenen Forschern je vielerei benannt. ${ }^{3,5,8,10}$

Wir nannten sie über-bzw. unterschiessende Oszillation. ${ }^{18}$

Diese Erscheinung scheint hauptsächlich von den Störungen des Kleinhirns und dessen Verbindungsbahn verursacht zu sein. Wir konnten sie bei zwei Fällen Marischer Degeneration, zwei Fällen der Blutung im Kleinhirn, einem Fall Kleinhirnhemisphärentumor,

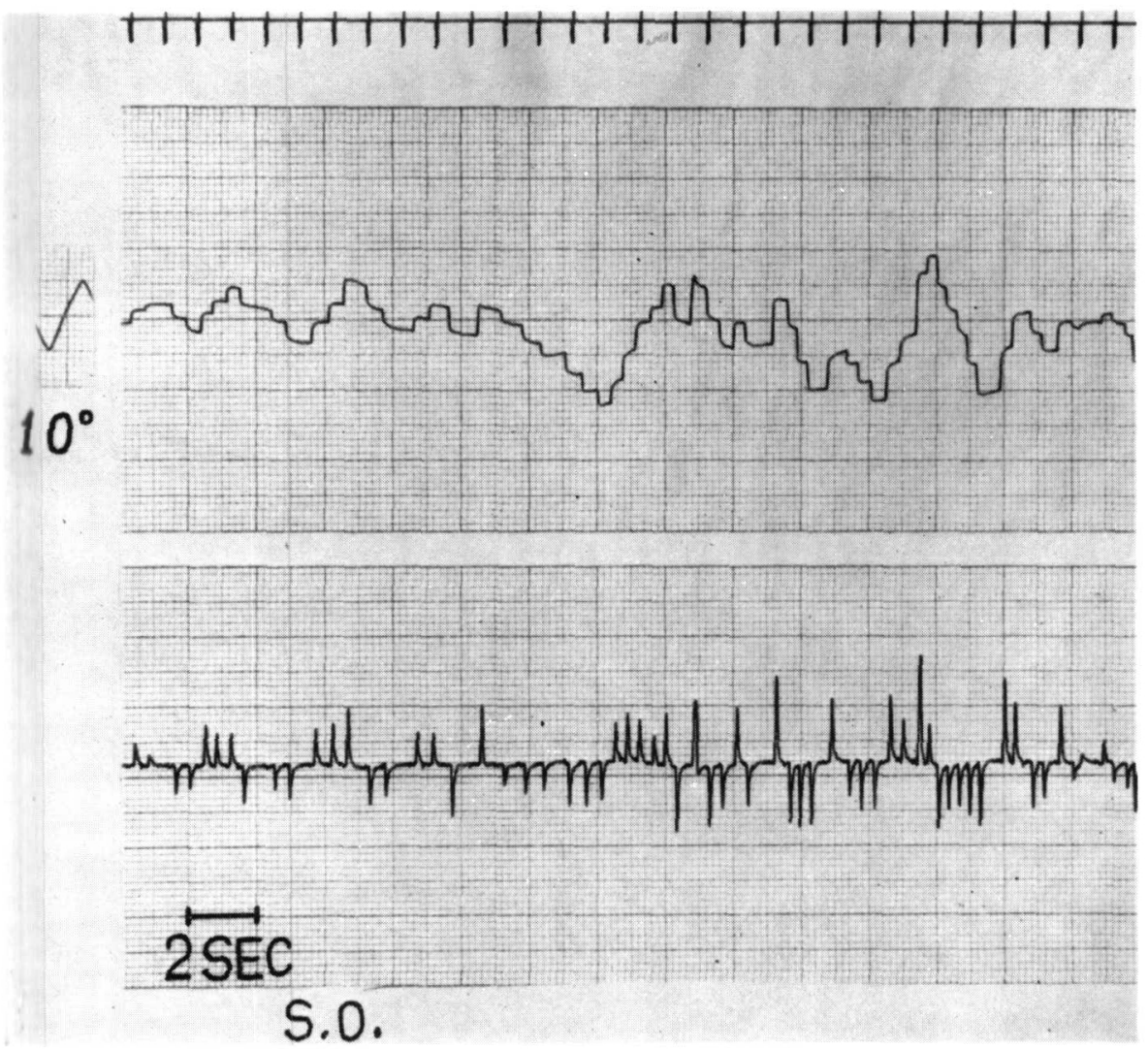

Abb. 1. S.O., 36 우

Die ENG-Registrierung der Fixationsrucke beim Medulloblastom. Die obere Kurve zeigt Augendeviation selbst mit Zeitkonstant von $3.0 \mathrm{sec}$ und die untere deren Geschwindigkeit mit Zeitkonstant $0.03 \mathrm{sec}$. Man muss die Fixationsrucke von Treppenrucken und Gegenrucken unterscheiden. Treppenrucke bei geschlossenen Augen sind eine seltene Variante der Gegenrucke: bei Registrierung mit kurzer Zeitkonstante bzw. der im Dunkelkammer können sie mit richtungswechselnder Spontannystagmus verwechselt werden. Gegenrucke und Treppenrucke sind normale Phänomene. 
einem Fall Medulloblastom, einem Fall Basilärer Impression, einem Fall Tumor des vierten Gehirnventrikels, drei Fällen Vertebro-Basilar-Insuffizienz sowie bei einem Fall Cerebellitis, insgesammt bei 12 Fällen beobachten.

Das Bild 2 ist die Registrierung eines Falls davon, und darin ist der Prozeß dargestellt, daß sich die Augapfelbewegung schwingend schwächt, wenn man den Blickpunkt nach rechts oder links wechselnd diese Bewegung plötzlich zum Stehen bringt.

3) Flügelschlägige Oszillation, flutter-like oscillation

Diese Erscheinung ist auch oft bei den Hinterschädelgrubenerkrankungen erkennbar und man kann, wie Higgins und Daroff ${ }^{9}$ erwähnen, nicht sagen, daß diese Erscheinungen nicht weniger sichtbar als die obengenannten über-bzw. unterschiessenden Oszillationen sind, und wenn man ausführlich und genau beobachtet, sind sie nicht selten zu betrachten. Auch wie sie sagen, ist die flügelschlägige Oszillation mehr bei Holmescher, Menzelscher oder OPCA-Degeneration als bei Friedreichscher Degeneration und multipler Sklerose erkennbar.

Nämlich, in dem Fall, daß der Spontannystagmus im engeren Sinne nicht sichtbar ist, läßt man den Patienten einen Blickpunkt um 30 Grad nach links bzw. rechts blicken (die Erscheinung des Blicknystagmus kommt dabei nicht in Frage), dann langsam in den Fixationszustand zurückversetzen. Dabei erscheint der Nystagmus (nystagmusartige

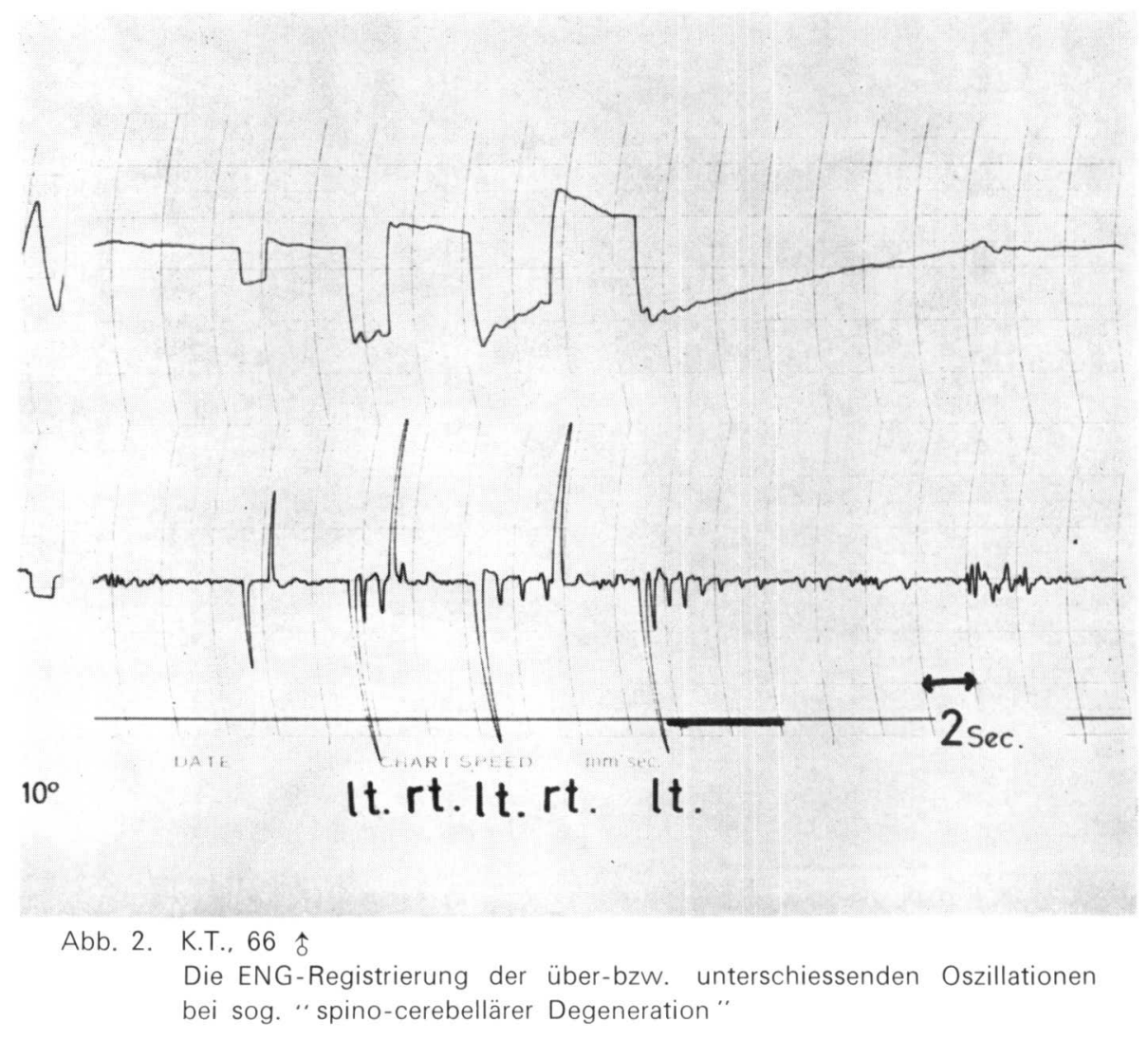


Bewegungen) mit Großschnelligkeitsphase in entgegengesetzter Richtung (Rückkehrrichtung) transitorisch. In diesem Fall sind diese Erscheinungen im allgemeinen bei den Fällen mit dem blickdysmetrischen Nystagmus besonders deutlich.

Das Bild 3 ist ein Beispiel davon. Im Zustand der Fixation ist der Nystagmus eigentlich nicht zu registrieren, aber bei der Zurückversetzung in den Fixationszustand nach dem Blick nach links oder rechts sind die transitorischen nystagmusartigen Bewegungen in entgegegesetzter Richtung deutlich registriert.

Wir gaben zuerst auf die Tatsahe, daß diese Erscheinung oft bei den Fällen des ruckartigen oder latenten Nystagmus unter den sogenannten "angeborenen Nystagmus"-Fällen erkennbar ist, acht. Durch diese Untersuchungen verstanden wir, daß diese Erscheinung auch bei den erworbenen Erkrankungen sichtbar ist. Wir behandelten bereits insgesammt 27 Fälle, d.h. spino-cerebelläre Degeneration; 9 Fälle, vaskuläre Störungen des Kleinhirens; 2 Fälle, vertebro-basilare Insuffizienz; 1 Fall, kongenitaler Nystagmus (ruckartiger bzw. latenter Nystagmus); 10 Fälle sowie traumatisches Halssyndrom; 5 Fälle.

Auch gerade nach der Operation des Kleinhirns ist sie oft zu erkennen. Das ist die
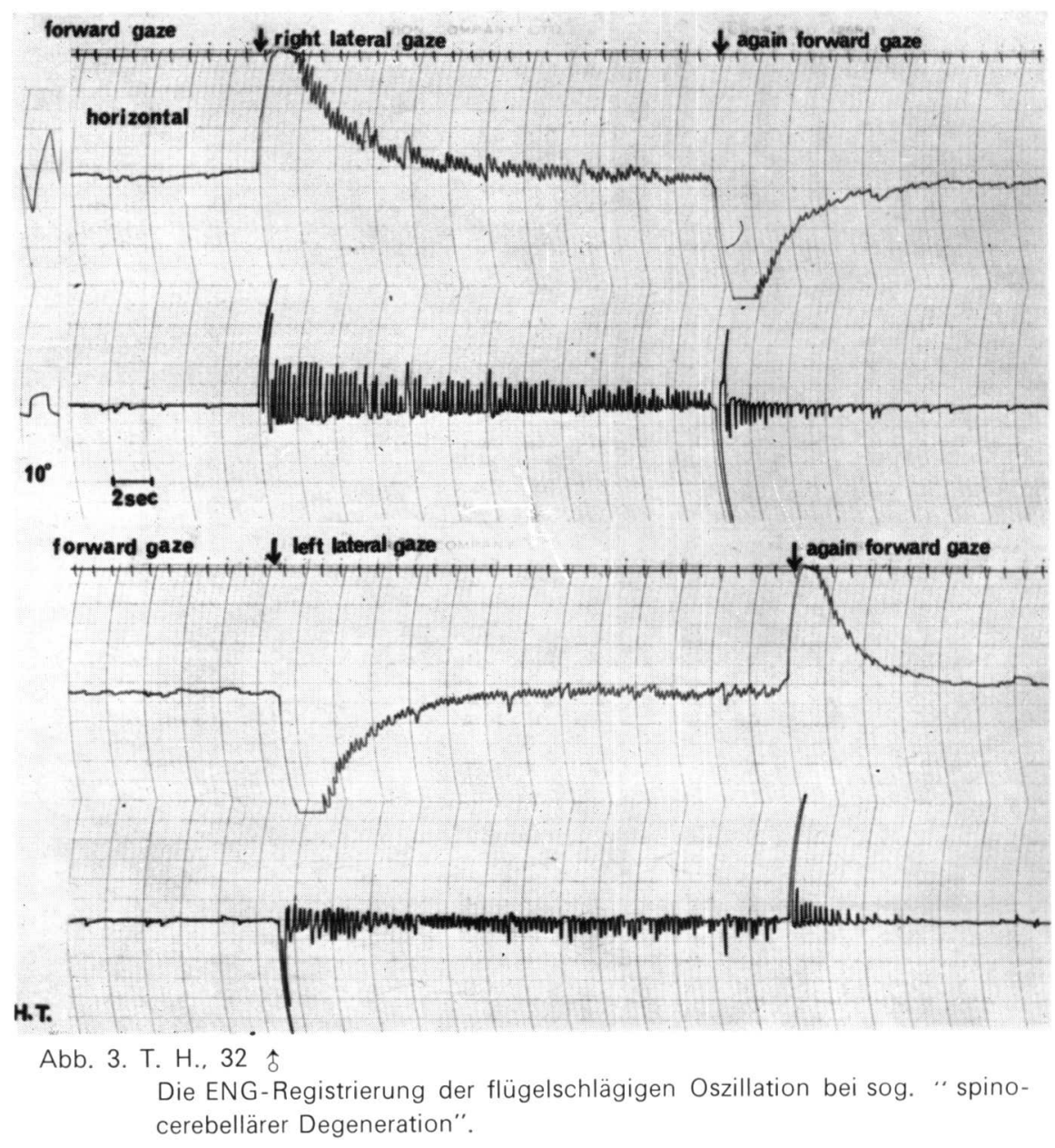

Equilibrium Res Suppl. 3 
a)

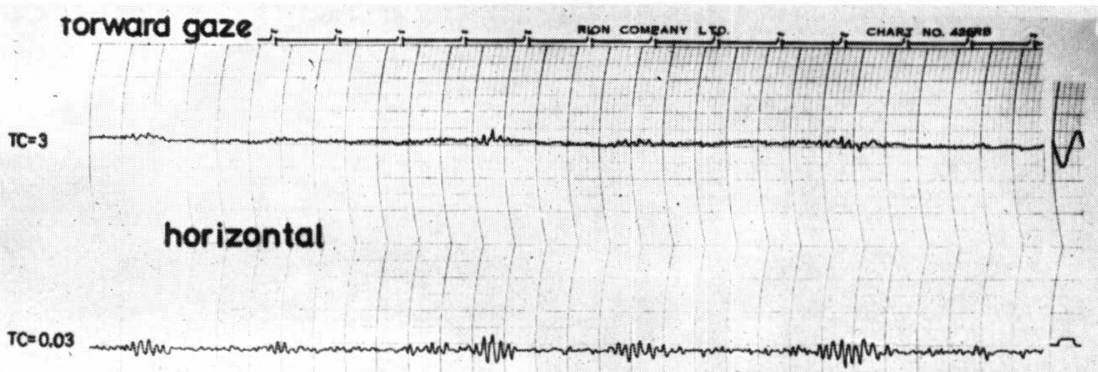

$$
1_{\text {sec. }}
$$

$\mathrm{TC}=3$

T.K. 540

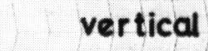

$\mathrm{TC}=0,03$

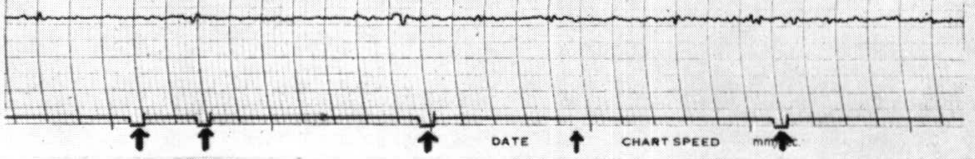

b)
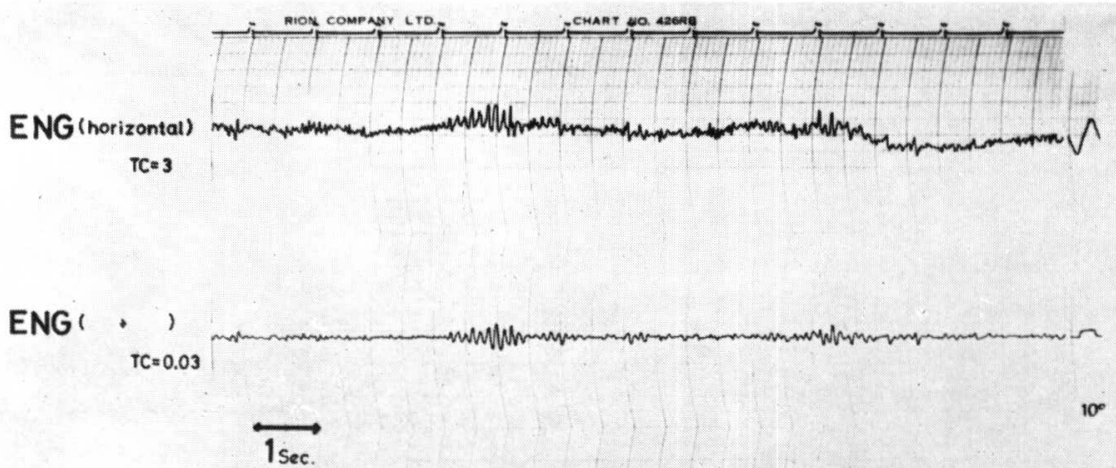

EEG

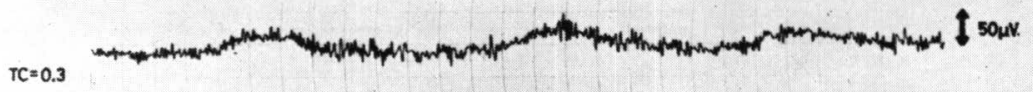

\section{ENG (vertical) \\ $\mathrm{TC}=0,03$}

T. K. 548

Abb. 4. T.K., 54 우

Die ENG-Registrierung der Blitzbewegungen bei Läsion des oberen Hirnstammes.

a) Horizontale und vertikale Ableitungen.

b) Horizontale und vartikale Ableitungen der Augenbewegungen und EEG. 
entwickelte Form der schon in den Artikel 1 und 2 erwähnten Phänomen anzunehmen.

Einige Forscher behaupten, daß die Benennung dieser flügelschlägige Oszillation für die im Artikel 2 erwähnte Erscheinung gilt, aber wir sind der Meinung, daß es gültig ist, die Erscheinung im Artikel 3 so zu benennen, wenn man verschiedene Erscheinungen auf den weiten Gesichtskreis systematisch ordnet. ${ }^{18}$

4) Blitzbewegungen, lightning eye movements

Es ist denkbar, daß diese Bewegungen hauptsächlich von den Extrapyramidalerkrankungen, besonders den Störungen im Tectoriumgebiet des Mesencephalons verursacht werden. Im allgemeinen sagt man, daß sie bei dem Tumor des Mittelhirns, Demyelisationskrankheit, multipler Sklerose usw. erkennbar sind, aber wir konnten sie bei 11 Fällen vaskulärer Mesencephlonläsion (Basilararteriesyndrom oder Syndrom der A. cerebelli superior), 3 Fällen anti-konvulsanter Mittelsvergiftung, 2 Fällen Tectalsyndrom des Mesencephalons, 1 Fall Tumor im hinteren Gebiet des dritten Gehirnventrikels, einem Fall Parkinsonsyndrom sowie 1 Fall traumatischem Halssyndrom, insgesammt bei 23 Fällen registrieren.

Bei denen krampft der Augapfel intermittierend, kurzzeitig, heftig nach rechts und links. Das ist nämlich der paroxysmale Krampf, der am Augenmuskel erscheint, der ist zwar hochfrequent, aber dauert meistens nur 1 bzw. 2 Sekunden und der Fall, der im Bild 4 gezeigt ist, bei dem der Krampf mehr als 2 Sekunden dauert, ist der Ausnahmefall.

Diese Bewegungen erscheinen unter allen Bedingungen, d.h. beim Augenöffnen, Augenschließen oder Augendecken, und sie sind im Vergleich mit der Fascikulation ein großer Ausbruch.

Man muß darauf aufmerksam sein, daß bei diesen Erscheinungen, die auch "in der Ruhe" (at rest) entstehen, verschiedene Kunstzeugnisse oft verkannt werden.

D.h. EMG oder EEG des Kaumuskels oder des Temporalmuskels werden häufig mißverstanden. Auf die Lidschlagbewegungen muß man auch achten. Deswegen bei der Registrierung dieser Erscheinungen registrierten wir gleichzeitig immer zum Vergleich die vertikalen Augenbewegungen oder wir führten EEG durch, um sie zu unterscheiden und richtig zu erkennen, doch wir meinen, wenn es zweifelhafte Registrierung gibt, sei es am wichtigsten, sich mit den eigenen Augen von der Existenz dieser Erscheinungen zu überzeugen, indem man sie gleichzeitig beobachtet (Bild 4, a-b.)

5) Opsoklonus, opsoclonus

Opsoklonus ist die konjugierte, schnelle, klonische und muskelkrapfhafte Augapfelbewegung und diese Bewegung ist über eine lange Periode reichend zu beobachten.

Sie ist sehr ruckartig, rythmisch und erscheint intermittierend massenweise, ferner beim Blick wird sie deutlicher, in diesem Punkt ist sie von der obengenannten Blickbewegung verschieden.

Opsoklonus verbindet sich mit der Ataxie und Myotonie des Musculus sceleti, aber dieser Opsoklonus dauert gewöhnlich am längsten und bleibt. Dessen Richtungen sind meistens horizontal und gelegentlich sind die Richtungen sichtbar, die rotierende und vertikale Neigung mithaben. ${ }^{12,13}$ Es gibt auch darunter Opsoklonus, dessen Frequenz 
ungefähr zehnmal pro Sekunde ist. Myotonie erscheint oft in derselben Periode mit der des Gaumen-, Mundhöhle-, Schlundkopf-oder des Kehlkopfmuskels.

Als Ursacheerkrankungen sind bisher Gehirnstammentzündung, vaskuläre Erkrankungen, hepatolentikulare Krankheiten und Tumor, ferner Kleinhirnentzündung denkbar. $7,12,18$

Wir konnten diese Erscheinungen bei 3 Fällen Hirnstammencephalitis, 1 Fall vaskulärer Mesencephlonläsion, 1 Fall Syringobulbie, 1 Falle Hirnstammepilepsie sowie 2 Fällen traumatischem Halssyndrom, insgesammt bei 9 Fällen registrieren (Bild 5).

Orzechowski ${ }^{13}$ und Gilbert ${ }^{7}$ meinen, daß sie von der Reizung bzw. Verheerung des $\mathrm{N}$. dentatus-Thalamus-Rindensystems verursacht sind, aber es ist genug denkbar, daß es eine pathologische Veränderung im dreieckigen Bereich zwischen N. ruber, N. dentatus und $\mathrm{N}$. olivae gibt, und einige Forscher sagen, daß die Pseudohypertrophie des N. olivae anatomisch besonders deutlich ist. ${ }^{12,22}$

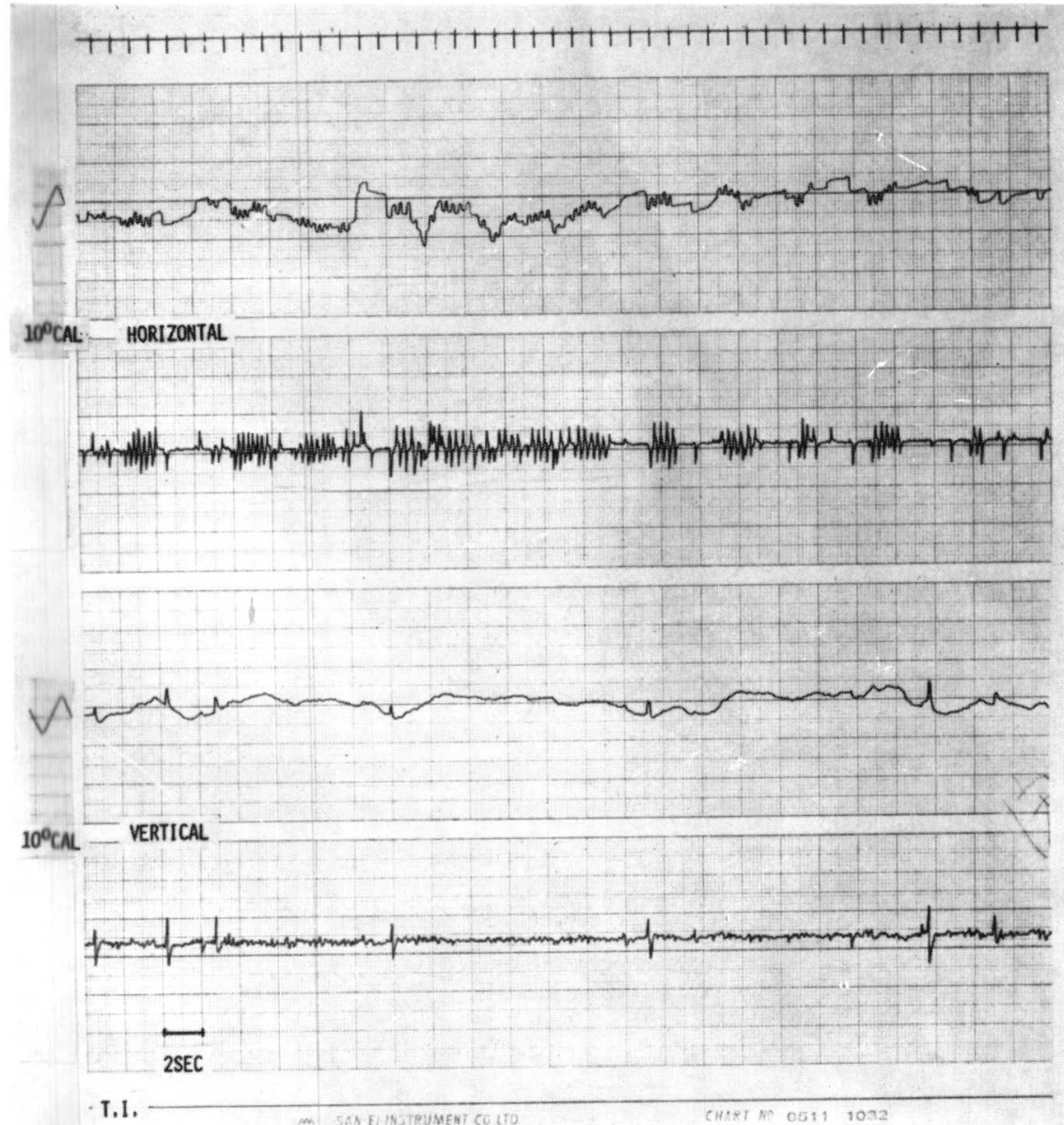

Abb. 5. T. I., 32 仓ิ

Die ENG-Rejgistrierung des Opsoklonus bei horizontalen und vertikalen Ableitungan bei vaskulärer Läsion des oberen Hirnstammes. 
6) Blickkrampf, gaze convulsion

Das ist die Erscheinung, daß sich der Augapfel heftig krampfhaft bewegt und plötzlich nach oben wendet, wenn der Patient einen Punkt blickt. Das ist nämlich der konjugierte Spasmus des Augenmuskels und dessen Richtung ist meistens nach Oberlid. Es geschieht gelegentlich, daß der tonische Krampf gleichzeitig am Gesicht, Körper und Gliedern mitbelgeitet wird. Diese Erscheinungen dauern einige Minuten, aber es gibt zuweilen die Fälle, bei denen sie etwa eine ${ }_{h}$ Stunde dauern.

Man sagt, daß der Blickkrampf bei der organischen Erkrankung erscheint und oft bei Encephalitis epidemica oder Parkinsonsyndrom sichtbar ist, und besonders gibt es die Erklärung, es sei durch die Reizung oder den Hemmungsverfall, die von der Vernarbung der Erkrankungen des Mittelhirns verursacht sind, erschienenes Symptom ${ }^{12}$.

Wir beobachteten ihn bei den folgenden 7 Fällen: 2 Fälle Tumor des dritten Gehirnventrikels, 2 Fälle Parkinsonsyndrom, 2 Fälle vaskuläre Mesencephlonläsion und ein Fall Pinealom.

7) Konvergenzkrampf, convergence convulsion

Die Konvergenz ist die Erscheinung, daß die beiden Blicke gleichzeitig nach innen devieren und zugleich der Augenstern verkleinert wird, wenn man einen vorstehenden Zeigepunkt blickt und währenddessen dieser Zeigepunkt immer näher verschoben wird.

Die Theories, daß diese Funktion hauptsächlich von den Rinden der beiden. Seiten repräsentiert wird und sich das subkortikale Zentrum in der prätektalen Region befindet, ist nicht festgesetzt. Seit langem ist es vermutet, daß es ein Konvergenzzentrum geben solle. Einige Forscher behaupten, daß das der Perliasche Kern ist, aber in diesen Punkt ist es noch nicht klar.

Der Konvergenzkrampf ist das Symptom, daß bei den Konvergenzbewegungen diese Bewegungen plötzlich mißfunktionieren oder wieder funktionieren. Über dieses Symptom berichtete Bielshowsky. ${ }^{2}$

Dieser Krampf wird oft von Blicklähmung nach oben oder Konvergenznystagmus bzw. retraktierendem Nystagmus kompliziert und ist bei dem Tumor im hinteren Gebiet des dritten Gehirnventrikels, dem Pinealom, der pathologischen Veränderung im Mittelhirn zu beobachten, aber man muß darauf achten, daß sich dieses Symptom auch bei Hysterie zeigen konnte.

Nicht nur bei der Konvergenz sondern auch beim Blick nach oben oder wenn dieser Blick nach oben dessen Grenze erreicht, ist es in dieser Erscheinung nicht selten, daß der Augapfel unter dem Zustand der Konvergenz zum Stillstand kommt.

Wir konnten dieses Symptom bei insgesammt 6 Fällen, d.h. Tumor des dritten Gehirnventrikels ( 3 Fälle), Pinealom (2 Fälle), vaskulärer Mesencephlonläsion (1 Fall) beobachten.

\section{ZUSAMMENFASSENDE BETRACHTUNGEN}

Es ist weit bekannt, daß bei den Störungen im Kleinhirn verschiedene "Kleinhirnsymptome" sichtbar sind. 
Hier möchte ich "Intentionstremor" als ein Beispiel solcher Symptome anführen und dadurch erklären. Beim Studium dieses Symptoms ist es selbstverständlich, ihn von dem gewähnlichen "Tremor" symptomatologisch oder auch bei der Begriffsbestimmung zu unterscheiden. Der Grund liegt darin, da $\beta$ der erstere eine Art der Ataxie zu schließen ist und deswegen er die Störung der konjugierten Bewegungen ist, während der letztere die regelmäßige Kontraktion des Synergistens oder Antagonistens ist.

Infolgedessen ist die Störung der konjugierten Bewegungen selbstverständlich unter dem Zustand der Ruhe nicht erkennbar und erscheint erst wenn man mit einer Absicht handelt. Dabei wird im allgemeinen die Schwingungsbreite der Bewegungen größer, wenn sie dem Endziel näher kommen.

Solche Erscheinung ist auch am Augenmuskel sichtbar.

Wir berichteten die Tatsache, daß beim Seitenblick der dysmetrische charakteristische Nystagmus erkennbar ist, der von dem blickparetischen Nystagmus bei den Hirnstammstörungen unterschieden ist, wenn es im Kleinhirn die Erkrankungen gibt. ${ }^{18}$

Wir konnten ferner bestätigen, daß es möglich ist, auch auf der frühen Stufe, wo solcher Nystagmus noch nicht erkennbar ist, charakteristische neurologische Symptome transitorisch $\mathrm{zu}$ verursachen, wenn eine einfache Belastung der Blickbewegung gegeben wird. Das sind die mit Beispielen angeführten dysmetrischen Augenbewegungen. Unserer Meinung nach entstehen diese Bewegungen alle auf Grund der Störungen des Kleinhirns sowie dessen Verbindungsbahn, und bei den Thalamusstörungen sind ähnliche Symptome über die Bahn des Thalmus/N. ruber/ und N. dentatus sichtbar.

Nämlich besitzt der Mensch die Funktion, das Stillstehende zu fixieren bzw. zu blicken und die Blickfolgefunktion nach dem sich bewegenden Ding.

An diesen Blickakommodationsbewegungen ist das okulomotorische System sehr beteiligt und ferner werden sie vom Impuls aus den Vestibulariskernen oder Gesichtsempfindungen, oder somato-sensoproprioceptiver Impuls aus den Halsrezeptoren und Klang beeinflußt. Die Beteiligung des Kleinhirns daran kann man nicht vernachlässigen.

Wenn das erstere partiell gestört wird, ist beim Seitenblick eines Punktes oft Nystagmus erkennbar. Der "blickparetische Nystagmus" ist als dessen repräsentatives Beispiel anzuführen, und bei Kleinhirnstörungen, besonders bei den tumorösen Erkrankungen kommen dazu ferner die Faktoren der konjugierten Bewegungsstörungen zwischen Augenmuskeln hinzu, die sich rechts und links je zu 6 Paar befinden, und der von uns behauptete "dysmetrische Blicknystagmus" erscheint.

Der letztere, d.h. die Funktionsstörung der Blickfolge des sich bewegenden Gegenstandes tritt oft, wie schon erwähnt, bei den Störungen des Kleinhirns sowie dessen Verbindungsbahn auf und deswegen erscheinen schon auf der frühen Stufe der Erkrankungen die charakterischen Symptome.

Bei der Fixation wird die schwache Bewegung der Fixation grob und noch dazu zeigen sich die ruckartigen Bewegungen mit pathologischer Richtung in den Augapfel. Das ist die "Fixationsrucke", die wir nannten. Diese Fixationstrucke wird bei den Hinterschädelgrubenerkrankungen beobachtet und hat eine bestimmte Beziehung mit der pathologischen 
Seite. Diese Bewegungen richten sich fast alle nach der wohlbefindenen Seite.

Diese Erscheinnung konnte D. Giorgio ${ }^{5}$ durch die Exstirpation des ganzen Kleinhirns des Hundes, Jung und Kornhuber ${ }^{11}$ bei den Fällen des beiderseitigen Kleinhirnerkrankungen finden.

Jedoch ist es.notwendig, solche Erscheinungen richtig zu erkennen, weil es physiologisch ähnliche Bewegungen gibt, nämlich, Gegenrucke oder Treppenrucke sind solche physiologischen Bewegungen; sie sind gewöhnlich nicht ruckartig, und auch wenn sie ruckartig sind, sind ihre Ruckartigkeit sehr schwach, ferner sind sie beim Augenöffnen in der Dunkelkammer, Augendecken, Augenschliessen, Narkose oder beim paradoxen Schlaf, auch beim Augenöffnen im Fall des alten Menschen, zu beobachten.

Die vertretenden dysmetrischen Augenbewegungen, die sich bei Belastung bestimmter Bewegungen zeigen, sind die von uns behauptete "über-bzw. unterschiessende Oszillation".

Sie wird oft als überschiessende Oszillation aufgefasst und man kann mit Recht sagen, das seien typische "Kleinhirnsymptome". Da bei fast allen Fällen des Kleinhirntumors oder der degenerativen Erkrankungen sie zu beobachten sind, und zwar auf der frühen Stufe, wo andere Symptome noch nicht sichtbar sind, könnten sie klinisch-diagnostisch ein wichtiger Schlüssel sein.

Beim Blick nach rechts oder links ist der horizontale oder rein rotierende sakkadierende Nystagmus am Patienten der Kleinhirnerkrankung oft erkennbar, aber es ist nicht selten, die ruckartigen Augenbewegungen in der zurückkehrenden Richtung bei der Zurückversetzung in den Fixationszustand transitorisch zu beobachten.

Diese Bewegungen sind gerade so wie "Flügelschlag des Schmetterlings" und wir nennen sie deshalb "flügelschlägige Oszillation".

Wir beachteten zuerst die Tatsache, daß bei den Fällen des ruckartigen kongenitalen Nystagmus oder latenten Nystagmus diese Erscheinungen mitbegleitet werden. Später erkannten wir, daß sie auch bei den erworbenen Erkrankungen, besonders bei den Kleinhirnerkrankungen oft zu beobachten sind. Unserer Meinung nach konnte das beim Studium der symptomatischen Physiologie des obengenannten kongenitalen Nystagmus ein sehr wichtiger Schlüssel sein.

Es ist schon allbekannt, daß bei den Extrapyramidalerkrankungen verschiedene unwirkürliche Bewegungen $\mathrm{zu}$ beobachten sind; Fasciculation, Myokymie, Myokolnus, Chorea, Athetose, Ballismus, Dystonie, Torticollis spasticus, Tremor, Intentionstremor, Spiegelbewegung usw. sind solche unwirkürliche Bewegungen.

Sie sind alle die unwirkürlichen Bewegungen, die hauptsächlich am Muskel in relativgrößer Einheit wie Gesicht, Körper oder Gliedermuskel und Gaumen erscheinen. Wenn eine dieser Erscheinungen zu beobachten ist, ist es wichtig, zur topischen Diagnose einen Anhalt zu finden, in welcher Gegend des extrapyramidalen Systems es die Störung gibt.

Dazu ist es notwendig, die Eigenschaften der pathologischen Bewegungen richtig zu analysieren und begreifen. Infolgedessen muß man auf die Lokalisation der Bewegungen, Umfang, Zustand der Muskelkontraktion, räumliche Regelmäßigkeit, zeitilchen Verlauf, Häufigkeit, Rhythmus, ferner auf Haltung, Empfindung, Schlaf, d.h. auf alle mögliche 
Bedingungen achten.

Über die Beziehung der verschiedenen unwirkürlichen Bewegungen mit der Herdlokalisation haben bisher viele Forscher ziemlich gründlich studiert und sie ist schon bekannt, aber es ist keine Übertreibung, zu sagen, daß das Studium von dieser Beziehung im Gebiet des Augapfels vernachlassigt ist. Unter gegenwärtigem Zustand sind die Berichte darübernämlich nur fragmentarisch, wie am Anfang dieser Abhandlung erwähnt.

Das Thema, das wir in dieser Abhandlung behandelten, ist gerade diese unwirkürlichen Bewegungen, die am Augapfel erscheinen. Sie sind oft mit den obengenannten heute weit bekannten unwirkürlichen Bewegungen kompliziert, oder es ist nicht selten, schon auf der frühen Stufe, wo andere Symptome noch nicht erkennbar sind, am Augapfel charakteristische Erscheinungen sichtbar zu sein, deshalb ist diese Erscheinung besonders bemerkenswert.

Unserer Meinung nach sind die Blitzbewegungen oder Opsoklonus auf Grund deren Erscheinungseigenschaften als klonischer Krampf zu begreifen, und wir sind der Meinung, daß es wahrscheinlich nichts dagegen sagen läßt. Die Blitzbewegungen sind oft bei der Vergiftung des antikonvulsanten Mittels zu beobachten, und das ist schon denkbar, wenn man die Lehre der Pharmakologie in Betracht zieht, daß solche Arznei den klonischen Krampf verursachen könnte.

Unter beiden Bewegungen, die als "klonische Augenbewegungen" eingeteilt sind, sind die Blitzbewegungen verhältnismäßig oft bei den Fällen der Erkrankungen am oberen Hirnstamm oder Diencephalonsystem sichtbar, während Opsoklonus ferner auch bei den Fällen der Erkrankungen am unteren Hirnstamm oder Kleinhirn zu beobachten ist. Es ist bemerkenswert und charakteristisch, daß die erstere nicht nur bei Fixation sondern auch unter dem Zustand der Ruhe ("at rest") erscheint, während die letztere besonders bei Fixation bzw. beim Blick beträchtlich erscheint.

Das ist auch ein großer Unterschied, daß sich die erstere nur am Augenmuskel zeigt, während von der letzteren auch am anderen Muskel die unwirkürlichen Bewegungen veranläßt werden.

Es ist sehr interessant und bemerkenswert, daß Holliday Myoklonus elektrophysiologisch wie folgt einteilte; Pyramidal-, Extrapyramidal- sowie lokalisiert segmentärer Spinalbzw. Bulbärmyoklonus.

Bei den als "tonische Augenbewegungen" eingeteilten zwei Bewegungen befinden sich die pathologischen Veränderungen im Tectorium des Mesencephalons oder in der vorderen Gegend, und es ist zu schließen, daß diese Erkrankungen bei der Erscheinung dieser Bewegungen die Hauptrolle spielen.

Weil sie immer beim Blick nach oben oder bei der Konvergenzbewegung erschieinen.

Nämlich, es gibt keine direkte Verbindung zwischen den Großhirnrinden und Augenmuskeln, sondern die beiden werden durch den subcorticalen prämotorischen Apparat des Nucleus intercalatus, d.h. durch Zwischenblickzentrum abgeschnitten, und diese Zentren haben verschiedene bedeutungsvolle Funktionen. Sie akkommodieren die der Blickrichtung oder Vergenzrichtung gemäß gestalteten konjugierten Bewegungen der beiden Augen 
und bringen verschciedene Blickakkommodationsbewegungen in Ordnung. Ferner ist es bekannt, daß sie die Funktion haben, ruckartige Augenbewegungen oder Nystagmusschnelligkeitsphase zu veranlassen. $\mathrm{Zu}$ den Blickzentren des Hirnstamms gehören ein Teil der Formatio reticularis medialis des Mesencephalons und Brückentectoriums, ferner auch einige der Kerne im Mesencephalon.

Das sind Cajalscher Nucleus interstitialis, Darkschewitschscher Kern und Hinterkommissurenkern. Infolgedessen, wenn im obengenannten Gebiet eine Störung verursacht wird, erscheint nicht einzelne motorische Störung eines Auges, sondern die konjugierte Blicklähmung (Parese).

Weil es zu schließen ist, daß das subcorticale Zentrum des vertikalen Blicks nach oben oder der Konvergenz im vorderen Gebiet des Tectoriums liegt. ${ }^{12}$

Auf jeden Fall werden diese pathologischen Augenbewegungen wahrscheinlich durch die Störungen der Nervenkerne des Extrapyramidalsystems, d.h. Corpus striatum, Globus pallidus, Nucleus subthalamicus, Nucleus niger, Nucleus ruber des Tectoriums, Nucleus dentatus, Tractus rubrospinalis, sowie durch die Störungen der Fasernverbindung zwischen diesen Kernen verursacht.

\section{ZUSAMMENFASSUNG}

Bei den Erkrankungen des Zentralnervensystems, besonders bei den verschiedenen Erkrankungen des Diencephalons oder im infratentorialen Gebiet spielt es klinisch-diagnostisch oft eine wichtige Rolle, die Eigenschaften der Augenbewegungen gründlich und sorgfältig zu untersuchen.

Die Kliniker haben jedoch allmählich größere Aufmerksamkeit auf den Nystagmus gerichtet, aber auf die spontanen pathologischen Augenbewegungen achten fast keine Forscher, so glauben wir.

In den letzten zwei Jahren beachteten wir diese Problem und die Zahl der Fälle, die durch die pathologische Anatomie und Operation, ferner durch die genauen neurologischen Untersuchungen diagnostiziert wurden, und bei denen diese Erscheinungen zu beobachten waren, beträgt 101 Fällen.

Auf Grund dieser Untersuchungen teilten wir sie wie folgt ein und ordneten sie systematisch; 1) dysmetrische Augenbewegungen, 2) klonische Augenbewegungen und 3) tonische Augenbewegungen.

Wir betrachteten die diagnostische Bedeutung der Lokalisation jedes Herdes sowie den Erscheinungsmechanismus und legen diese Untersuchungen zur Kritik vor.

\section{SUMMARY}

Precise analysis and study of the characteristics of eye movements are diagnostically paramount means to know various diseases, which are found in the central nervous system, especially in the subtentorial area or in the diencephalon.

However, up to now many a clinician has paid much attention to the nystagmus; they almost always pay no attention to spontaneous pathological eye movements except

Equilibrium Res Suppl, 3 
the nystagmus.

We have paid much attention and have studied the problem for the last two years, there were 101 cases found with the non-nystagmic spontaneous pathological eye movements, which were verified by autopsy and/or surgical operation and/or precise neurological examinations.

According to the results we gained, the non-nystagmic spontaneous pathological eye movements are classified arranged and settled down as follows: 1) dymetric eye movements (a: fixation jerks, b: over-or undershoot oscillation, $c$ : flutter-like oscillation), 2) myoclonic eye movements (a: lightning eye movements, b: opsoclonus), 3 ) tonic eye movements (a: gaze convulsion, $\mathrm{b}$ : convergence convulsion)

In addition, we mentioned the pathophysiology and the topic diagnostic significance of each non-nystagmic spontaneous pathological eye movements.

\section{DANKWORT}

Zum Schluß möchte ich hier Herrn Prof. Shozo Kawamura meine Dankbarkeit für seine freundliche Anleitung und Revision dieser Abhandlung bezeigen. Ich danke auch meinen Mitarbeitern, Mitgliedern der Forschungsgruppe der Otoneurologie der UnivHNO-Klinik. Die Mitarbeit von Frl. Dipl. scie. Kuniko Kinoshita sowie Yoko Yamaguchi werde ich nicht vergessen. Den Hauptinhalt dieser Abhandlung habe ich in der 13. Jahrestagung der klinischen Neurologie Japans (Sendai) sowie in der 73. Japanischen Generalversammlung für HNO-Ärzte (Gifu) veröffentlicht.

Diese Arbeit wurde von der Takeda-Forschungsstiftung finanziell unterstützt.

\section{LITERATUR}

1) BaCh-Y-Rita, P. \& Collins, C. A.: The control of eye movements, 343, Academic Press, New York, 1971.

2) Bielshowsky, A.: Lecture on motor anomalies of the eyes. III. Paralysis of the conjugate ocular movements of the eyes. Arch. Ophthal. (Chicago) 13:569-583, 1935.

3) Cogan, D. G.: Brain lesions and eye movements in man. (Bender, M. B.: The oculomotor system, Hoeber, New York, 417-423, 1964).

4) Cogan, D. J.: Brain lesions and eye movements in man. (Walsh, G. B. \& HoYT, W. F.: Clinical Neuro-Ophthalmology, Williams \& Wilkins, Baltimore, Vol. 3, 215-247, 1969).

5) Di Giongio, A.: Reflexes vestibularies et cervelet. Acta oto-rhino-laryng. belg. 4: 282, 1950.

6) Frenzel, H. H.: Spontan-und Provokations-Nystagmus als Krankheitssymptome, Springer, Berlin-Göttingen-Heidelberg, 1955.

7) Gilbert, G. J., Mc Entee, W. J. III \& Glaser, G. H.: Familial myoclonus and ataxia. Neurology (Minneap.) 13: 365-372, 1963.

8) Goldstein, J. E. \& CogAn, D. G.: Lateralizing value of ocular motor dysmetria and skew deviation. Arch. Ophth. (Chicago) 66: 517-518, 1961.

9) Higgins, D. C. \& Daroff, R. B.: Overshoot and oscillation in ocular dysmetria. Arch. Ophth. (Chicago) 15: 742-745, 1966.

10) Holmes, G.: The symptoms of acute cerebellar injuries due to gun shot injuries. Brain 40: 461-537, 1917.

11) JunG, R. \& KorNhuber, H. H.: Results of Electronystagmography in man: The value of optokinetic, vestibular and spontaneous nystagmus for neurologic diagnosis and research. (Bender, M. B.: The oculomotor system, Hoeber, New York, 428-481, 1964).

12) Kornhuber, H. H.: Physiologie und Klinik des zentralvestibulären Systems (Blick-und Stütz- 
motorik). HNO-Heilkunde, B. III/Teil 3, Georg Thieme, Stuttgart, 2151-2351, 1966.

13) Orzechowski, C.: De Látaxic dysmétrique des yeux. Remarques sur látaxie des yeux dite myoclonique (opsoclonie, opsochorie). J. Psychol. Neurol. 35: 1, 1927.

14) Sakata, E. \& Komatsuzaki, A.: Spontan-und Provokations-Nystagmus. Seine diagnostische Bedeutung bei Gleichgweichtsstörungen mit und ohne Schwindel. HNO (Berl.) 14, 289-298, 1966.

15) Sakata, E.: Das experimentelle Studium über den Spontan-und Provokations-Nystagmus. Das Studium I: Das Zerstörungsexperiment der einseitigen Kaninchen-Labyrinth und des Kleinhirns. Jap. Jour. Otol. Tokyo 70:385-394, 1967.

16) Sakata, E. u.a.: Das neurootologische Studium über die Kleinhirnstörung. Der I. Bericht: Ob die Störung der Kleinhirnhemisphäre durch die Prüfung der Augenbewegung diagnostiziert werden kann? Pract. Otol. (Kyoto) 61: 1642-1658, 1968.

17) Sakata, E. u.a.: Der Kleinhirntumor und Schwindelanfall, Ménière Krankheit und deren Umkreis, V. Bericht. Pract. Otol. (Kyoto) 62: 1089-1100, 1969.

18) Sakata, E.: Wie kann man die Gleichgewichtsstörungen durch die Untersuchung des spontanen pathologischen Augenbewegung mit und ohne Leuchtbrille diagnostizieren? Pract. Otol. Kyoto 63: 431-463, 1970.

19) Sakata, E.: Neurootologisches Studium über die Läsion des Kleinhirnwurms. Equilibrium Res. Suppl. 1 : 30-48, 1971.

20) Sakata, E. et al: Diagnostic importance of spontaneous pathological eye movement. Equilibrium Res. Suppl. 2, 50-63, 1972.

21) Sakata, E.: Pathohistologische Befunde eines sezierten klinischen Falls des periodisch-alternierenden horizontalen Nystagmus (N. alternans), im Druck.

22) SCHENK, E.: Hirnnervenmyorhythmie, ihre Pathogenese und ihre Stellung im myoklonischen Syndrome. Monog. Neurolog. Psychia. 109, Springer, Heidelberg, 1965.

(Received April 3, 1972) 